\title{
ANATOMICAL SPECIMENS FROM PRESERVED ANIMAL DISCARDS
}

At the end of the term, laboratory instructors dispose of dissected specimens that are often in excellent condition. We have developed a simple technique for permanently hardening bone-muscle preparations and preserving certain other organs. The preparations are more durable and life-like than most commercial models.

Specimens preserved in formalin, alcohol, or embalming fluid can be preserved by this technique. Select an anatomical unit, such as a bisected head, entire foreleg, hind limb, shoulder region, or thigh, and dissect the desired muscles from origin to insertion. We find that isolation of three or four muscles produces detail and simple beauty that is most desirable. If injected blood vessels and large nerves are left intact, they can contribute significantly to the finished product. The quality of the preparation varies directly with the quality of the dissection.

After the connective tissue, fat, and skin are thoroughly removed, soak the preparation in acetone (technical grade) for approximately two weeks. It appears that specimens can be stored indefinitely in the acetone without damage. Due to the degreasing and dehydrating properties of acetone, it should be changed if it becomes cloudy or discolored. Care should be taken to suspend the preparation in the acetone rather than letting it touch the sides or bottom of the container. Since the acetone shrinks the muscles, it may be necessary prior to soaking to put pieces of sponge or cloth between the muscles to keep them separated. In a few instances it is necessary to mount the specimens in a frame.

After removing the specimen from the acetone, allow it to dry for 15-20 minutes. Too long a drying time causes dessication, and the preparation becomes too dark. Bathe the dried preparation in heated (60 $\left.{ }^{\circ} \mathrm{C}\right)$ casting resin with catalyst (15 drops/liter). The resin, which can be purchased along with the catalyst at most paint supply stores, may be poured over the preparation, or the preparation may be immersed in the resin. After the specimen is thoroughly coated, suspend it by a wire in a warm, well-ventilated room to dry. Tackiness will disappear in two or three days. Throughout the drying period, secure the muscles in the desired orientation with pins or wood swabs. If bubbles appear on the surface of the bones or muscles they can be removed by brushing the affected area lightly with acetone. Finally, apply one or two coats of clear semi-gloss lacquer over the dry, resinated specimen for a more pleasing and protective finish. Specimens shown in the figure were prepared from embalmed cats, embalmed human cadavers, and an alcohol-preserved sheep heart.

The advantages of this preparation are numerous. Specimens are more life-like than commercial models; thus, students can more easily compare their dissections to them. In situations such as audiotutorial teaching, where wet specimens are difficult to use, the preparations are an excellent substitute. For the practical examination, tape pointers can be affixed to the preparations and questions can be made up at the convenience of the instructor. The

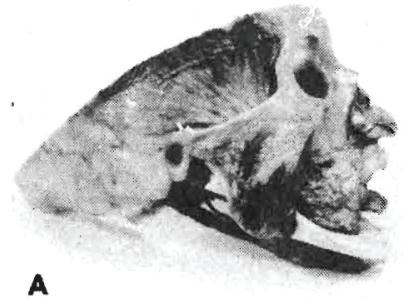

A

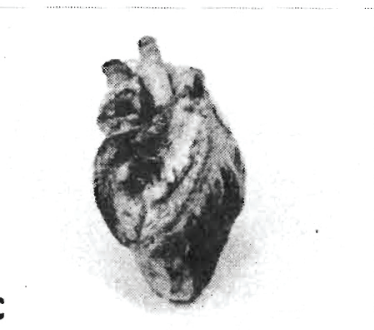

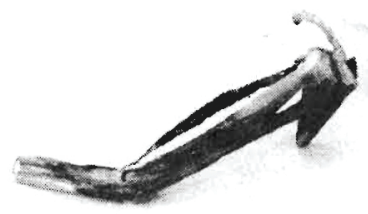

B

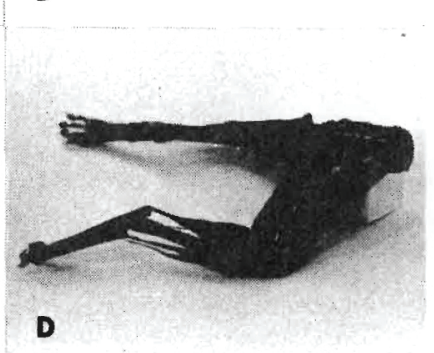

Anatomical preparations produced by the acetone-resin technique. A. Bisected human head. B. Human arm and forearm. C. Sheep heart. D. Cat hind limb. 
acetone-resin technique is more easily executed and less time-consuming than other techniques that produce quality preparations (Hildebrand 1968). These durable preparations are easily repaired with epoxy resin; and dirty or marred surfaces can be washed off with water, dried, and then relacquered. Finally, with the acetone-resin technique, excellent anatomical specimens can be prepared at a fraction of the cost of commercial models.

Acknowledgment.-A Creative and Research Effort (CARE) Grant was awarded to Harry L. Fierstine by California Polytechnic State University for the perfection of the acetone-resin technique. This research would not have been possible without this grant-in-aid.

\section{Reference}

HrLDEBRAND, M. 1968. Anatomical preparations. University of California Press, Berkeley.

Harry L. Fierstine, Myron A. Amerine, and Kathleen L. Bek

Biological Sciences Department

California Polytechnic State University San Luis Obispo, Calif. 93407 